\title{
Cardiotoxicity with immune system targeting drugs: a meta-analysis of anti-PD/PD-L1 immunotherapy randomized clinical trials
}

\author{
Mohamed Rahouma*,‡,1, Nagla Abdel Karim,2, Massimo Baudo³, Maha Yahia4, Mohamed \\ Kamel $^{1}$, Ihab Eldessouki ${ }^{2}$, Ahmed Abouarab ${ }^{3}$, Ihab Saad ${ }^{1}$, Adham Elmously ${ }^{3}$, Katherine D \\ Gray $^{5}$, Galal Ghaly ${ }^{1}$, Ola Gaber ${ }^{2}$, Mona Kamal ${ }^{6}$, Ayah A. Hassan, Mostafa Rahouma ${ }^{8}$, \\ Fabrizio D'Ascenzo ${ }^{9}$, John Morris², Abdelrahman Mohamed ${ }^{1}$, Leonard Girardi ${ }^{3}$ \& Mario \\ Gaudino $^{3}$ \\ ${ }^{1}$ Surgical Oncology Department, National Cancer Institute, Cairo University, Egypt \\ ${ }^{2}$ Medical Oncology Department, University of Cincinnati Cancer Institute, Cincinnati, OH 45220, USA \\ ${ }^{3}$ Cardiothoracic Surgery Departments, Weill Cornell Medicine, New York, NY 14853, USA \\ ${ }^{4}$ Medical Oncology Department, National Cancer Institute, Cairo University, Egypt \\ ${ }^{5}$ Department of Surgery, New York Presbyterian Hospital, Weill Cornel Medicine, New York, NY 14853, USA \\ ${ }^{6}$ Radiation Oncology Department, MD Anderson Cancer Center, Huston Texas, TX 77030, USA \\ ${ }^{7}$ Department of Pharmacognosy, Faculty of Pharmacy, Cairo University, Egypt \\ ${ }^{8}$ Information Technology Department, National Cancer Institute, Cairo University, Egypt \\ ${ }^{9}$ Department of Cardiology, Città della Scienza e della Salute, University of Turin, Turin, Italy \\ *Author for correspondence: mhmdrahouma@gmail.com \\ $¥$ Authors contributed equally
}

Background: With antiprogrammed death receptor-1 (anti-PD-L1) therapy, a recent meta-analysis reported higher incidence of cutaneous, endocrine and gastrointestinal complications especially with dual anti-PD-L1 immunotherapy (IMM). Methods: Our primary outcome was assessment of all cardiotoxicity grades in IMM compared with different treatments, thus a systemic review and a meta-analysis on randomized clinical trials (RCTs) were done. Results: We included 11 RCTs with 6574 patients (3234 patients in IMM arm vs 3340 patients in the other arm). Three non-small-cell lung cancer RCTs, seven melanoma RCTs and only one prostatic cancer RCT met the inclusion criteria. There were five RCTs that compared monoimmunotherapy to chemotherapy " $(\mathrm{n}=2631$ patients)". No difference exists in all cardiotoxicity grades or high-grade cardiotoxicity $(p>0.05)$. Lung cancer exhibited a higher response rate and lower mortality in IMM. Conclusion: There was no reported statistically significant cardiotoxicity associated with anti-PD/PD-L1 use. Lung cancer subgroups showed better response and survival rates.

First draft submitted: 16 August 2018; Accepted for publication: 6 March 2019; Published online: 15 May 2019

Keywords: anti-PD/PDL $1 \bullet$ cardiotoxicity $\bullet$ chemotherapy $\bullet$ immune system targeting drugs $\bullet$ immunotherapy $\bullet$ lung cancer $\bullet$ meta-analysis $\bullet$ randomized clinical trials

In the era of immunotherapy, specifically, immune-checkpoint blocking antibodies as antiprogrammed death receptor-1 (anti-PD/PD-L1) and anticytotoxic T lymphocyte-associated antigen 4 (anti-CTLA-4) used in various malignancies, mainly melanoma, non-small-cell lung cancer (NSCLC) and renal cell carcinoma, a plethora of immune-mediated adverse events have been frequently encountered. In previous case series and meta-analyses, a higher incidence of cutaneous, gastrointestinal and endocrine complications was reported with anti-PD-L1 therapy and some even reported cadiotoxicity. Consequently, a higher incidence with dual anti-PD-L1 therapy was reported [1-4]. In this study, our aim was to determine if anti-PD/PD-L1 had higher incidence of cardiotoxicity compared with other treatment protocols.

\section{Methods}

Search strategy and study selection of this systematic review were conducted according to the Preferred Reporting Items for Systematic Reviews and Meta-Analyses (PRISMA) guidelines [5]. In February 2017, the PubMed, 
MEDLINE and EMBASE databases were searched for publications containing anti-PD/PD-L1 immunotherapy (IMM) by the words 'Atezolizumab' OR 'Avelumab' OR 'BMS936559' OR 'Durvalumab' OR 'Ipilimumab' OR 'Nivolumab' OR 'Pembrolizumab' OR 'Pidilizumab' OR 'Tremelimumab' that were obtained from a previously published review [6]. All studies comparing mono-immunotherapy versus other single/multiple treatments and reporting any cardiac complications were identified. Cardiac complications included arrhythmias as ventricular tachycardia and atrial fibrillation, pericardial effusion, pericarditis, myocardial infarction, cardiac myositis, cardiomyopathy, death from cardiac arrest or acute heart failure [4].

The bibliography of all studies and any related meta-analyses were searched to identify further articles that potentially could be recruited, in other words, backward snowballing.

Inclusion criteria for the present analysis were: cancer-related, Phase (II/III), randomized clinical trials (RCTs) on humans published in English language with availability of full article that reports any previously mentioned cardiotoxicity.

In case of absence of monoimmunotherapy in one treatment arm, the study was excluded. In case of multiple publications from the same center or overlapping between studies, only the publication with the largest sample size was considered. Non-English studies, studies on animals, case reports, review articles, editorials and expert opinions were also excluded.

Two authors (M Baudo and M Rahouma) independently inspected the electronic reports identified by the searches. In case of discrepancies, they were resolved by the third author (M Gaudino) opinion and consensus meeting.

The quality of included studies was assessed using The Cochrane Collaboration's tool for assessing risk of bias in RCTs [7] (Supplementary figure 1).

\section{Study outcomes}

Cardiotoxicity grades in IMM in comparison to other treatments were our primary end point. The secondary end points were: comparing cardiotoxicity grades in IMM to others in lung cancer and subgroups; the cardiotoxicity grade difference in between IMM and other studies having lung cancer and other cancer subgroups and in IMM compared with chemotherapy (CTH)-only studies; outcome of IMM compared with others (in all studies and in lung cancer/other cancers subgroups) as well as in IMMs compared with CTH only studies; and difference in mortality in the same groups. High-grade adverse events were defined as grade 3 or higher identified by National Cancer Institute Common Terminology Criteria for Adverse Events (NCI-CTCAEv.4) [8] that means Severe, Life-threatening and Death for grade 3, 4 and 5, respectively.

\section{Data extraction \& statistical analysis}

Microsoft Office Excel 2010 (Microsoft, Redmond, Washington) was the used program for data extraction. Continuous variables are expressed as median (25th, 75 th percentile) or as mean \pm standard deviation. Categorical variables are reported as (\%).

Study design, study period, country, study center, trial Phase (II/III), cancer type, comparison arms, doses of drug administered, inclusion/exclusion criteria, treatments arms and sample size were documented. The following patient characteristics were registered: age, sex, smoking, Eastern Cooperative Oncology Group performance status (ECOG-PS), stage, cardiotoxicity complications (see earlier; all grade/high grade), response (complete and partial responders were considered as responders) and all-cause mortality.

All-cause mortality was derived from Kaplan-Meier overall survival curves at the end of follow-up using a previously described method [9-11] with aid of GetData Graph Digitizer software 2.26 (http://getdata-graph-digiti zer.com/). Relative ratios (RR) with $95 \%$ confidence intervals (95\% CI) for cardiotoxicity events were calculated by means of the DerSimonian-Laird (inverse variance [IV]) method [12]. Exploiting the assumption that HRs are asymptotically similar to relative risks, we calculated relative risks according to the formula described by Grant $e t$ al. [13]. Random-effect model was used for statistical outcome pooling, computing risk estimates with CI and in case of heterogeneity $\left(\mathrm{I}^{2}\right)>25$, otherwise, fixed model was used.

Funnel plots were used for assessment of publication bias by graphical inspection. We have set the hypothesis testing for equivalence using the two-tailed 0.05 level. The hypothesis testing for statistical homogeneity was setting the two-tailed 0.10 level. Cochran $Q$-test was used as a base with $I^{2}$ values of 25,50 and $75 \%$. (each of those value show mild, moderate and severe homogeneity) 


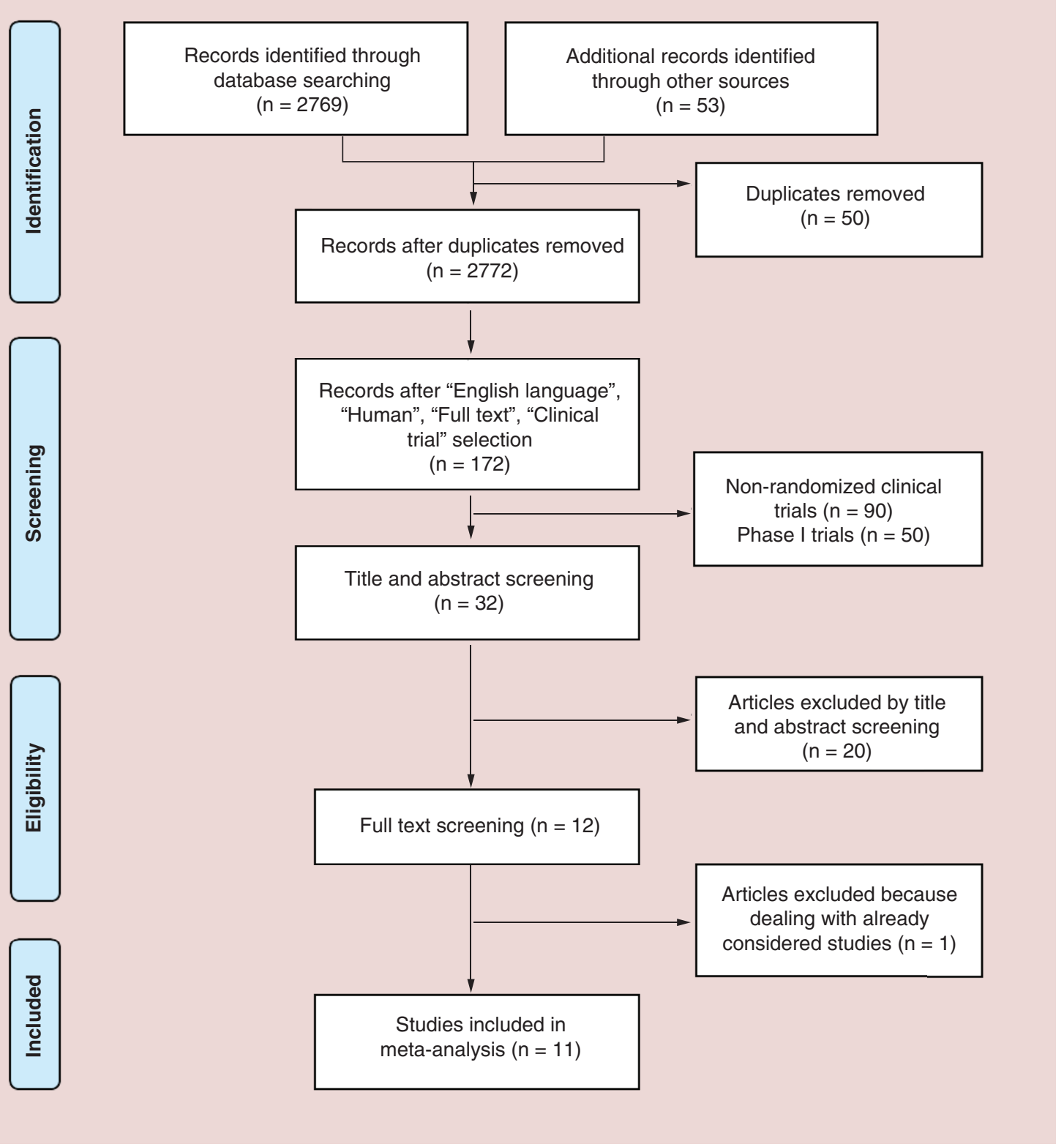

Figure 1. PRISMA flowchart of our recruited articles. Among 2769 searched articles from database searching and 53 additional articles, 11 articles met our inclusion criteria.

We used Review Manager version 5.3 to do this meta-analysis and comprehensive meta-analysis version 2 for sensitivity analysis using 'one study removed analysis'.

\section{Results}

\section{Eligible studies \& characteristics of studies}

Figure 1 shows an outline of the systematic review process. We identified 2822 studies for clinical outcomes, which ended at 2772 studies after removal of duplicates. We also assessed 32 full text articles for eligibility. Eleven RCTs met our inclusion criteria, we included 6574 patients. We found 3234 (49.19\%) patients in IMM arm and in the other arm we had 3340 (50.88\%) patients. We analyzed three NSCLC RCTs that included 1588 patients, subclassification of those showed 782 in IMM arm and 776 in other arm. Moreover, seven RCTs were included for melanoma patients $(n=4190)$, and only one RCT for prostate cancer that had been included according our inclusion 


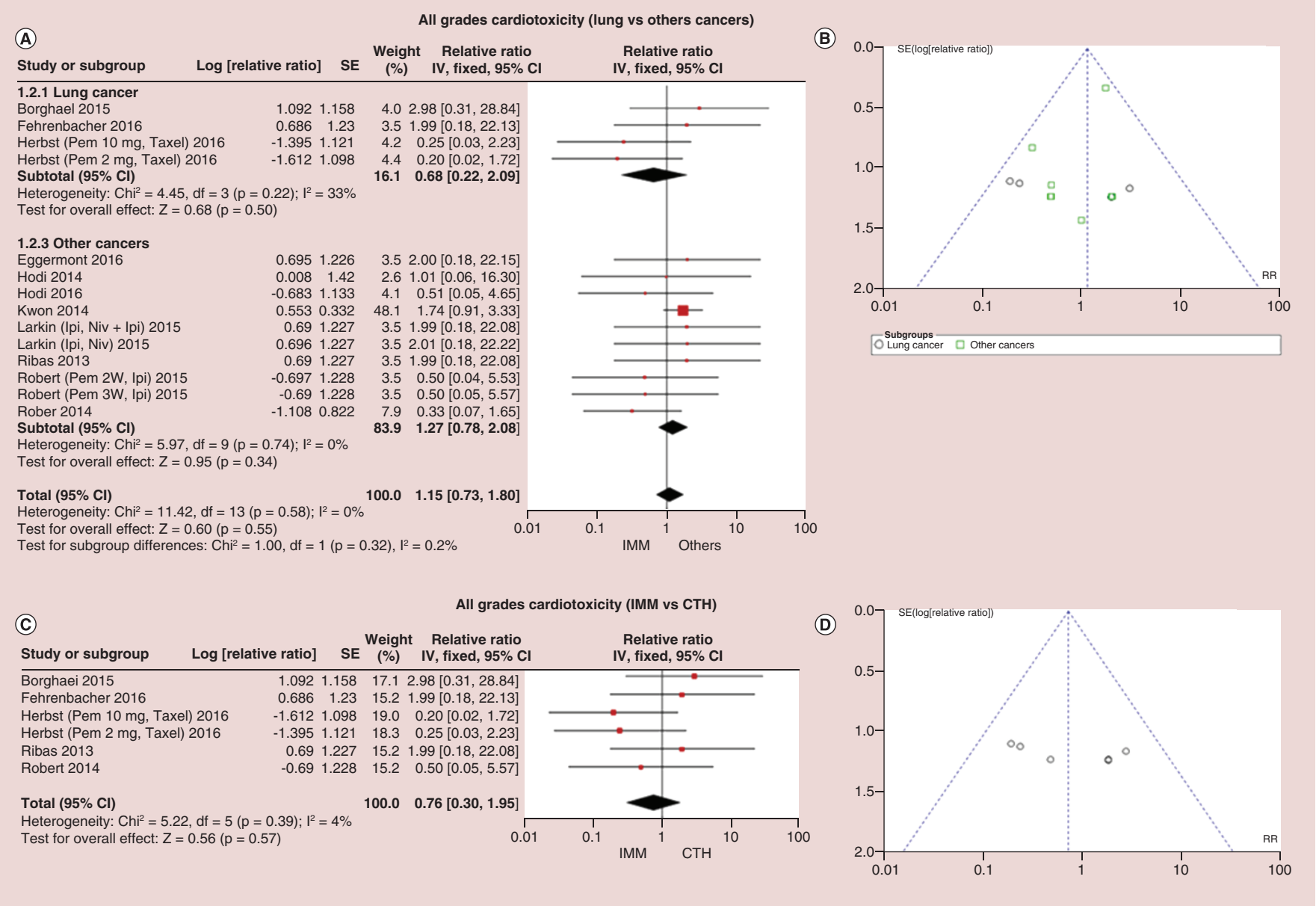

Figure 2. Forest \& Funnel plots of all-grade cardiotoxicity in different subgroups. (A \& B) Forest \& Funnel plots of lung versus other cancers studies. (C \& D) Forest \& Funnel plots of immunotherapy versus chemotherapy-only studies.

criteria. In addition to the above-mentioned studies, we included five RCTs that compared monoimmunotherapy to CTH (four studies used docetaxel, one study used temozolomide or dacarbazine), those studies had 2631 patients, subclassification showed 1320 in IMM arm and 1311 patients in CTH arm. The pooled mean follow-up was 18.69 and 18.52 months in IMM and other arm, respectively. Criteria of the included studies are shown in Table 1 and Supplementary Table 1.

\section{Meta-analysis of the outcomes \\ Cardiotoxicity in immunotherapy}

There was no difference in all grades cardiotoxicity among all recruited studies (RR: 1.15; 95\% CI: 0.73-1.80; $\mathrm{p}$ $=0.55)$ or in subgroups of lung cancer (RR: $0.68 ; 95 \%$ CI: $0.22-2.09 ; \mathrm{p}=0.50)$ or other cancers $(\mathrm{RR}=1.27$; 95\% CI: 0.78-2.08; $\mathrm{p}=0.34$ ) (Figure 2). One study removed analysis for all grades cardiotoxicity was shown in Supplementary Figure 1. Similarly, no difference in high grades cardiotoxicity among all recruited studies (RR: 1.47; 95\% CI: 0.87-2.46; $\mathrm{p}=0.15$ ) or in subgroups of lung cancer (RR: $0.80 ; 95 \% \mathrm{CI}: 0.25-2.50 ; \mathrm{p}=0.70$ ) or other cancers (RR: 1.71, 95\% CI: 0.96-3.06; p = 0.07) (Supplementary Figure 3; Table 2).

\section{Response in immunotherapy}

No difference in response between comparative arms (IMM vs others) could be identified (RR: 1.41; 95\% CI: $0.91-$ 2.17; $\mathrm{p}=0.12$ ). While lung cancer showed a higher response rate (RR: $1.65 ; 95 \% \mathrm{CI}: 1.27-2.15 ; \mathrm{p}=0.0002$ ), no statistically significant difference in response regarding other types of cancers could be identified but heterogeneity exists $(\mathrm{I} 2=93 ; \mathrm{p}<0.0001)$. On restricting studies to those comparing response in IMM versus CTH only, 


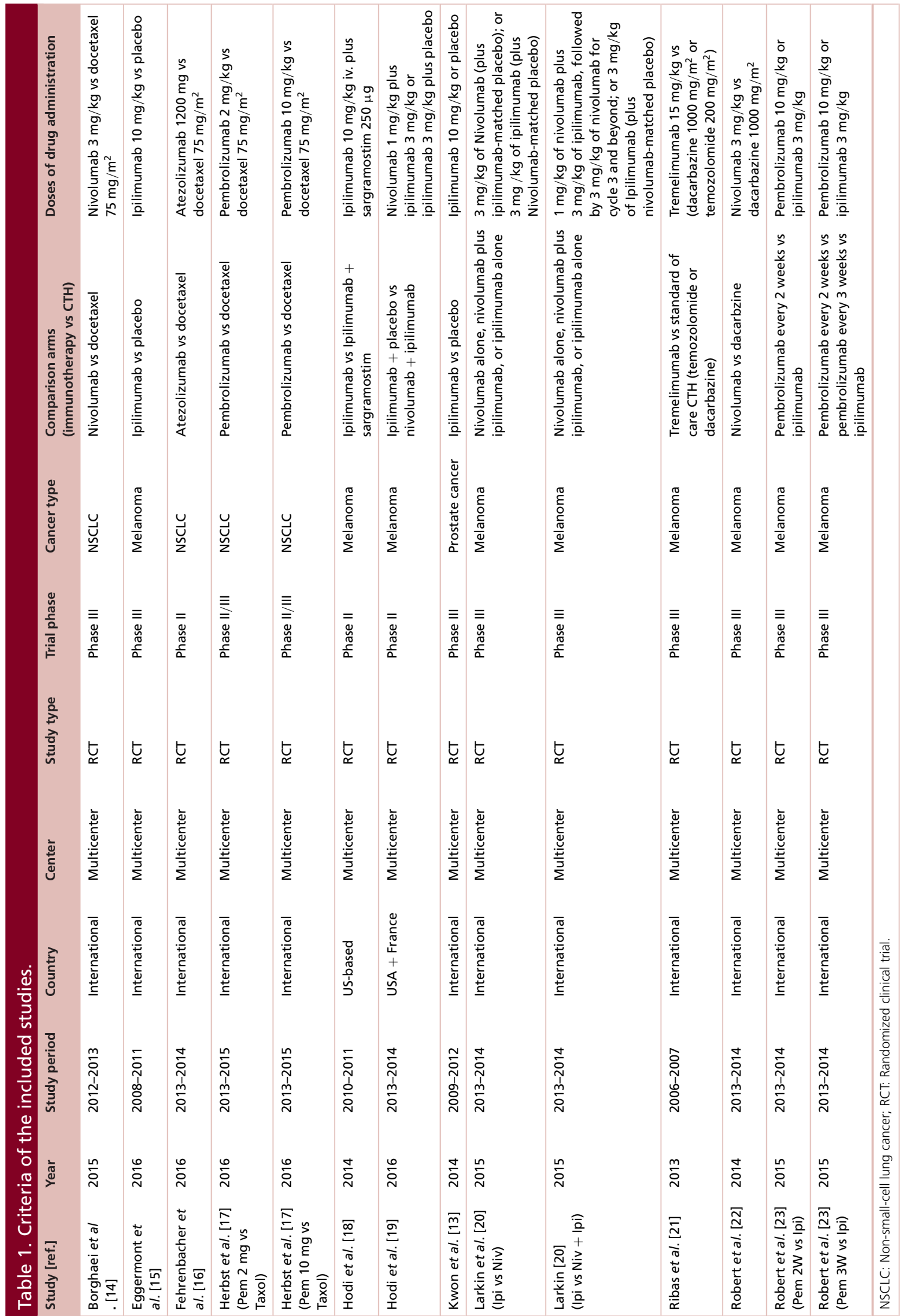


Table 2. Summary of outcomes among anti PD-L1 immunotherapy recruited studies.

\begin{tabular}{|c|c|c|c|c|c|}
\hline Outcome or subgroup & Studies & $\begin{array}{l}\text { Effect estimate (RR; } \\
95 \% \mathrm{Cl})\end{array}$ & $\begin{array}{l}\text { Heterogeneity }\left(I^{2}\right) \text {, } \\
\text { p-value }\end{array}$ & Overall effect & Favors \\
\hline \multicolumn{6}{|l|}{ I. Cardiotoxicity in immunotherapy } \\
\hline All grades cardiotoxicity (All studies) & 11 & $1.15(0.73,1.80)$ & $I^{2}=0 ; p=0.58$ & $Z=0.60 ; p=0.55$ & None \\
\hline \multicolumn{6}{|c|}{ All grades cardiotoxicity in immunotherapy (lung vs others subgroups) } \\
\hline - Lung cancer & 3 & $0.68(0.22,2.09)$ & $I^{2}=33 ; p=0.22$ & $Z=0.68 ; p=0.50$ & None \\
\hline - Other cancers & 8 & $1.27(0.78,2.08)$ & $\mathrm{I}^{2}=0 ; p=0.74$ & $Z=0.95 ; p=0.34$ & None \\
\hline $\begin{array}{l}\text { All grades cardiotoxicity in immunotherapy (IMM vs CTH } \\
\text { only) }\end{array}$ & 5 & $0.76(0.30,1.95)$ & $1^{2}=4 ; p=0.39$ & $Z=0.56 ; p=0.57$ & None \\
\hline High-grade cardiotoxicity (all studies) & 11 & $1.47(0.87,2.46)$ & $\mathrm{I}^{2}=0 ; \mathrm{p}=0.72$ & $Z=0.45 ; p=0.15$ & None \\
\hline \multicolumn{6}{|c|}{ High-grade cardiotoxicity in immunotherapy (lung vs others subgroups) } \\
\hline - Lung cancer & 3 & $0.80(0.25,2.50)$ & $I^{2}=14 ; p=0.32$ & $Z=0.39 ; p=0.70$ & None \\
\hline - Other cancers & 8 & $1.71(0.96,3.06)$ & $\mathrm{I}^{2}=0 ; p=0.85$ & $Z=1.82 ; p=0.07$ & None \\
\hline $\begin{array}{l}\text { High-grade cardiotoxicity in immunotherapy (IMM vs CTH } \\
\text { only) }\end{array}$ & 5 & $0.95(0.36,2.50)$ & $\mathrm{I}^{2}=0 ; \mathrm{p}=0.56$ & $z=0.10 ; p=0.92$ & None \\
\hline \multicolumn{6}{|l|}{ II. Response in IMM } \\
\hline Response in IMM vs others & 10 & $1.41(0.91,2.17)$ & $I^{2}=90 ; p<0.0001$ & $z=1.55 ; p=0.12$ & None \\
\hline Response in lung vs other cancers & 10 & $1.41(0.91,2.17)$ & & & \\
\hline - Lung cancer & 3 & $1.65(1.27,2.15)$ & $I^{2}=13 ; p=0.33$ & $Z=3.74 ; p=0.0002$ & IMM \\
\hline - Other cancers & 7 & $1.32(0.71,2.45)$ & $I^{2}=93 ; p<0.0001$ & $Z=0.87 ; p=0.38$ & None \\
\hline Response in IMM vs CTH & 5 & $1.68(1.25,2.26)$ & $I^{2}=53 ; p=0.06$ & $Z=3.46 ; p<0.001$ & IMM \\
\hline \multicolumn{6}{|l|}{ III. OS at end of follow-up } \\
\hline OS at end of follow-up (all studies) & 10 & $0.84(0.69,1.02)$ & $\mathrm{I}^{2}=59 ; \mathrm{p}=0.005$ & $Z=1.77 ; p=0.08$ & None \\
\hline OS at end of follow-up (lung vs other cancers) & 10 & $0.84(0.69,1.02)$ & & & \\
\hline - Lung cancer & 3 & $0.80(0.65,0.97)$ & $\mathrm{I}^{2}=0 ; p=0.83$ & $Z=2.24 ; p=0.03$ & IMM \\
\hline - Other cancers & 7 & $0.86(0.63,1.16)$ & $I^{2}=72 ; p<0.001$ & $Z=1.00 ; p=0.32$ & None \\
\hline OS at end of follow-up (IMM vs CTH) & 5 & $0.73(0.56,0.95)$ & $\mathrm{I}^{2}=58 ; p=0.04$ & $Z=2.35 ; p=0.02$ & IMM \\
\hline
\end{tabular}

immunotherapy arm showed a higher response rate (RR: 1.68; 95\% CI: 1.25-2.26; p < 0.001) (Figure $3 \&$ Table 2).

\section{End of follow-up overall mortality}

There was no statistically significant difference in the end of follow-up overall mortality in immunotherapy versus others in all recruited studies (RR: 0.84; 95\% CI: 0.69-1.02; $\mathrm{p}=0.08$ ), but heterogeneity exists $\left(\mathrm{I}^{2}=59 ; \mathrm{p}=0.005\right)$. On subgroups analysis, patients received immunotherapy showed lower mortality versus the other arm in lung cancer (RR: 0.80 ; 95\% CI: 0.65-0.97; $\mathrm{p}=0.03$ ), but this was not statistically evident in the other included types of cancer (RR: 0.86; 95\% CI: 0.63-1.16; $\mathrm{p}=0.32)$ but heterogeneity exists $\left(\mathrm{I}^{2}=72 \% ; \mathrm{p}=0.0007\right)$. After including IMM versus CTH studies only, lower mortality was evident in IMM arm (RR: 0.73 ; 95\% CI: 0.56-0.95; $p=0.02$ ) (Figure 4).

Outcomes summary is shown in Table 2.

\section{Discussion}

Up-to-date and up-to-our knowledge, this is the first meta-analysis reporting cardiotoxicity outcomes in immunotherapy.

With the current era of immunotherapy in cancer treatment, we aimed to assess the cardiotoxicity associated with anti-PD/PD-L1 use that included arrhythmias, pericardial effusion or inflamation, myocardial infarction, cardiac myositis, cardiomyopathy, death from cardiac arrest or acute heart failure. Four Prior RCT reported two cases of cardiac arrest and two cases of cardio-respiratory arrest in Ipilimumab $10 \mathrm{mg} / \mathrm{kg}$ arm when compared with placebo-related death [16] and another one reported acute cardiac failure-related mortality in Atezolizumab $1200 \mathrm{mg}$ when compared with Docetaxel $75 \mathrm{mg} / \mathrm{m}^{2}$ [17]. Myocardial infarction death was reported in Herbst $e t$ al. three arm trial when they compared Pembrolizumab $10 \mathrm{mg}$ versus docetaxel arms but not in Pembrolizumab 


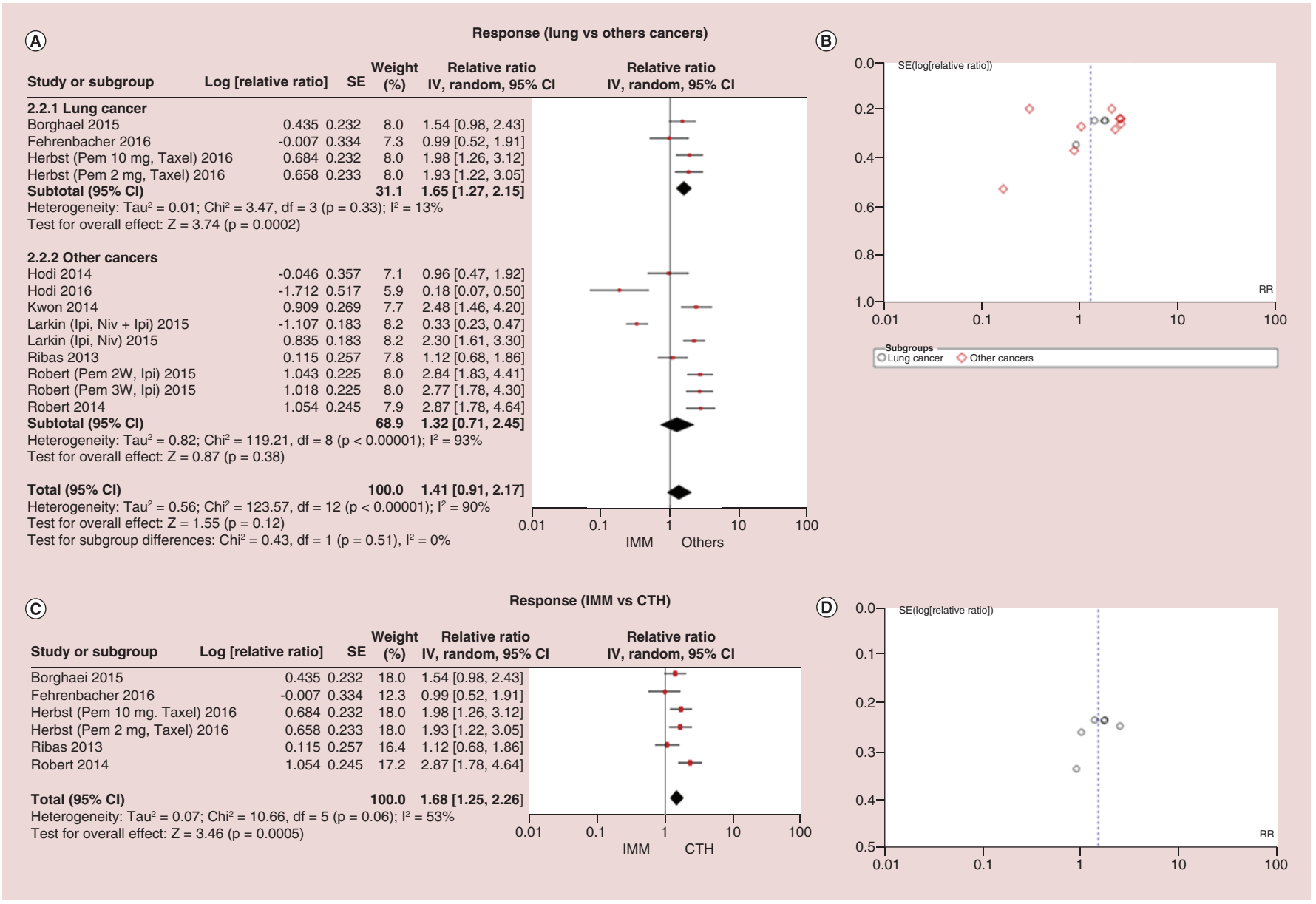

Figure 3. Forest \& Funnel plots of response in different subgroups. (A \& B) Forest \& Funnel plots of lung versus other cancers studies. (C \& D) Forest \& Funnel plots of immunotherapy versus chemotherapy-only studies.

$2 \mathrm{mg}$ versus docetaxel arms [14,15]. So, we aimed to pool many studies to identify the actual incidence and properly estimate the risk of those apparently rare events with such effective treatments. No difference in all/high grades cardiotoxicity was evident in our meta-analysis.

No difference in response except after comparing IMM versus CTH only and in RCTs on lung cancer patients. Although individual studies showed better response to IMM but pooling of several studies with heterogeneous pathology omit this response difference; however, this statistical difference re-appears after isolation of NSCLC as a separate subgroup analysis as outcomes drawn from lung cancer reported RCTs were generally homogenous (the highest I 2 in all reported outcomes was 33\%) and elaborate the previously confirmed results of several recruited $[14,17,24]$ or unrecruited RCTs [15] in this meta-analysis that IMM was associated with higher response and survival.

This meta-analysis met its end point of providing an evidence for absence of significantly higher horrific cardiotoxicity mentioned by some of the published RCTs as mentioned earlier.

Being the first meta-analysis reporting cardiotoxicity outcomes in immunotherapy represents an inherited strength in our study; however, among the limitation of our meta-analysis is that it included heterogeneous types of cancers (three NSCLC RCTs, seven melanoma RCTs, one prostate cancer RCT) and heterogeneous second treatment arm (CTH, dual IMM, Sargramostim and placebo); however, in order to make it more homogenous, we did several subgroup analyses.

\section{Conclusion}

Despite the fact that anti-PD/PD-L1 previously showed greater incidence of complications including gastrointestinal, cardiotoxicity was statistically insignificant regardless the treatment regimen either chemotherapy or dual 


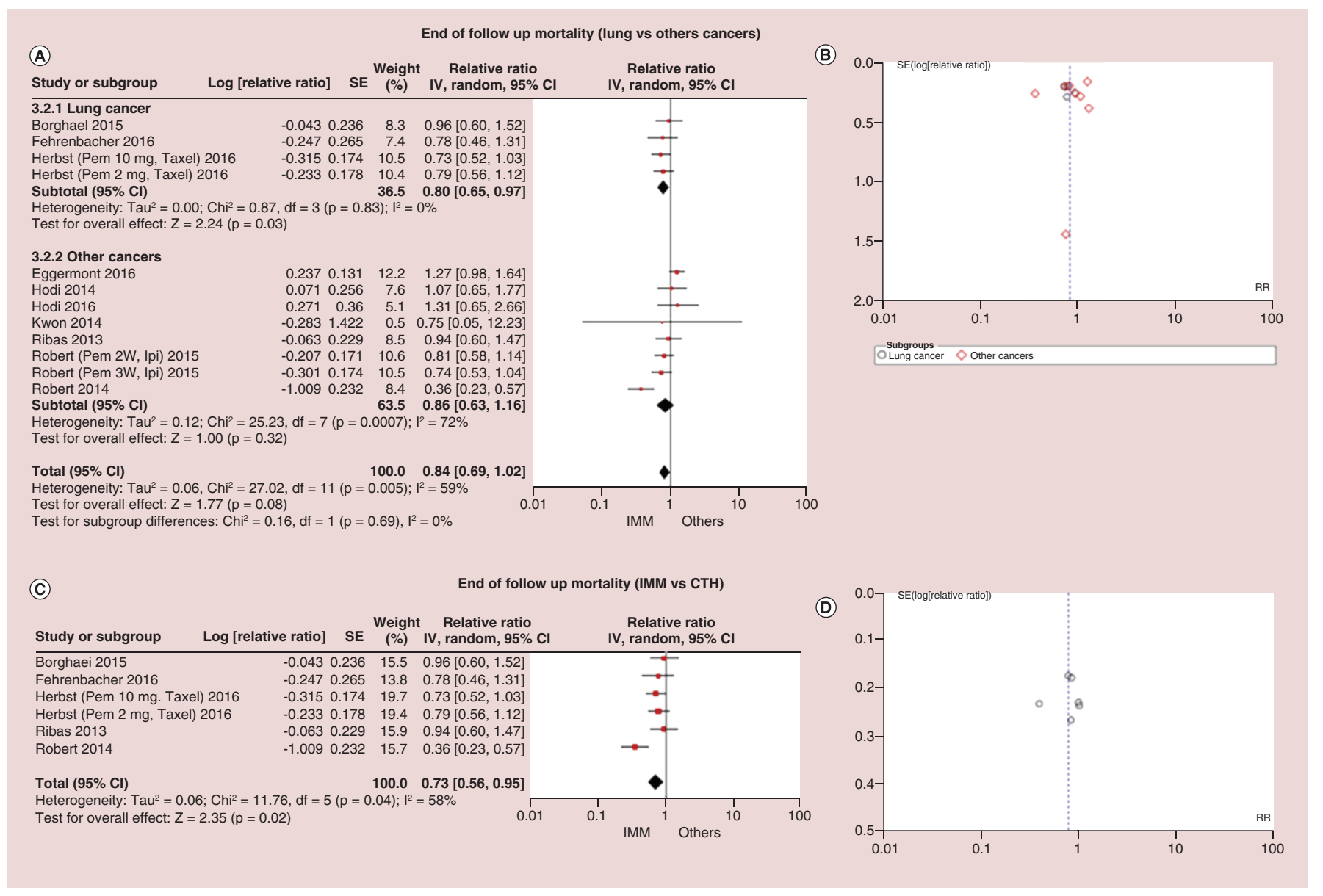

Figure 4. Forest \& Funnel plots of overall mortality at end of follow-up in different subgroups. (A \& B) Forest \& Funnel plots of lung versus other cancers studies. (C \& D) Forest \& Funnel plots of immunotherapy versus chemotherapy-only studies.

immunotherapy. The use of anti-PD/PD-L1 provided better response and survival rates when used in lung cancer subgroup.

\section{Future perspective}

Due to the efficacy of anti-PD/PD-L1 and anti-CTLA- 4 in treatment of various malignancies, growing number of patients are assigned to this line of treatment. Devastating as it may seem, cardiotoxicity due to these drugs has to be detected and managed as early as possible. The involvement of cardiologists in the initial planning for treatment or in the early cardiac dysfunction assessment is not a standard of care yet [25], that is why a multidisciplinary approach is recommended, including oncologist and an expert cardiologist for early identification and management of cardiotoxicities. Despite being a specific and sensitive marker of cardiotoxicity, troponin-I is not routinely monitored in most immunotherapy trials [26,27]. We recommend inclusion of thorough cardiac monitoring in the treatment plan, including assessment of troponin level, routine electrocardiograms, as well as echocardiographs to enhance our knowledge of the real incidence of early and late cardiotoxicities associated with immune checkpoint inhibitors. Nevertheless, clear validated guidelines for management of checkpoint inhibitors-associated myocarditis are urgently needed through prospective studies.

While various grades of cardiotoxic events were described with the use of immune checkpoint inhibitors including myocarditis, heart failure, heart block, myocardial fibrosis and cardiomyopathy, the exact mechanism of such complications is not fully understood. Johnson et al. [28] described two cases of fulminant myocarditis and myositis associated with combination of ipilimumab plus nivolumab where histological analysis showed that the myocardium, the cardiac sinus and the atrioventricular nodes were infiltrated by lymphocytes, and macrophages. Also, high expression of PD-L1 was demonstrated on affected cardiomyocytes and on infiltrating $\mathrm{CD} 8^{+} \mathrm{T}$ cells. 
Thus, we may conclude that cardiac toxicities that arise with the use of immune checkpoint inhibitors are mostly immune mediated. In previous reports, these toxicities were successfully managed using systemic high dose steroids [29,30]. Based on this, we recommend exploring the role of low dose steroids in decreasing immune checkpoint inhibitors cardiotoxicities in future prospective studies.

\section{Executive summary}

\section{Background}

- Immune-mediated adverse events have been frequently encountered with the use of antiprogrammed death receptor-1 (anti-PD/PD-L1) and anticytotoxic T lymphocyte-associated antigen 4 in treatment of various malignancies.

- These adverse effects include cutaneous, gastrointestinal, endocrine complications as well as various degrees of cardiotoxicities.

- We aimed to determine if there was a higher incidence of cardiotoxicity with the use of anti-PD/PD-L1 compared with other treatment protocols.

\section{Methods}

- We searched the PubMed, MEDLINE and EMBASE databases for publications containing anti-PD/PD-L1 immuotherapy (IMM) by the words 'Atezolizumab' OR 'Avelumab' OR 'BMS936559' OR ‘Durvalumab' OR 'Ipilimumab' OR 'Nivolumab' OR ‘Pembrolizumab' OR 'Pidilizumab' OR 'Tremelimumab' that were obtained from a previously published review [6].

Study outcome

- The primary end point was all grade difference in cardiotoxicity in IMM versus other treatments.

- The secondary end points were:

- The difference of all cardiotoxicity grades in IMM in comparison to others in lung cancer, in addition comparing IMM to CTH.

- Comparing cardiotoxicity higher grades in between IMM (within different cancer subgroups) and IMM to CTH.

- Outcome of IMM compared with others (in all studies and in lung cancer/other cancers subgroups) as well as in IMMs compared with CTH only studies.

- Difference in mortality in the same groups.

Results

Meta-analysis of the outcomes

Cardiotoxicity in immunotherapy

- Regarding all grades cardiotoxicity, no difference was found in all recruited studies $(p=0.55)$ or subgroups of lung cancer $(p=0.50)$ or other cancers $p=0.34)$.

- There was no difference in high grades cardiotoxicity among all recruited studies $(p=0.15)$ or in subgroups of lung cancer; $(p=0.70)$ or other cancers $(p=0.07)$.

Response in immunotherapy

- There was no difference in response between comparative arms (IMM vs others) $(p=0.12)$.

- Lung cancer showed a higher response rate $(p=0.0002)$.

- There was no statistically significant difference in response regarding other types of cancers, but heterogeneity exists $(p<0.0001)$.

- Immunotherapy arm showed a higher response rate on restricting studies to those comparing response in IMM versus CTH only $(p<0.001)$.

Discussion

- This is the first meta-analysis reporting cardiotoxicity outcomes in immunotherapy, up-to-date and up-to-our knowledge.

- Our aim was to assess the cardiotoxicity associated with anti-PD/PD-L1 use.

- There was no evident difference in all/high grades cardiotoxicity in our meta-analysis.

- No difference in response could be identified except after comparing IMM versus CTH only and in RCTs on lung cancer patients.

- This statistical difference reappears after isolation of non-small-cell lung cancer as a separate subgroup analysis.

- We met our end point in this meta-analysis by proving the absence of IMM associated higher cardiotoxicity rates mentioned by some of the published RCTs.

Conclusion

- Anti-PD/PD-L1 previously showed higher incidence of cutaneous, gastrointestinal, endocrine complications.

- There was no statistically significant cardiotoxicity was associated with anti-PD/PD-L1 in contrast to other treatment regimens. 
Since human and murine hearts express PD-L1 $[31,32]$ and the fact that many experiments suggested that PD1/PD-L1 and CTLA-4 play important roles in limiting T cell-mediated autoimmune myocarditis [33-39], we suggest further research to find a cardioprotective drug against the decrease in cardiac PD-1/PD-L1 and CTLA-4 levels.

\section{Financial \& competing interests disclosure}

The authors have no relevant affiliations or financial involvement with any organization or entity with a financial interest in or financial conflict with the subject matter or materials discussed in the manuscript. This includes employment, consultancies, honoraria, stock ownership or options, expert testimony, grants or patents received or pending, or royalties.

\section{Open access}

This work is licensed under the Attribution-NonCommercial-NoDerivatives 4.0 Unported License. To view a copy of this license, visit http://creativecommons.org/licenses/by-nc-nd/4.0/

\section{Supplementary data}

To view the supplementary data that accompany this paper please visit the journal website at: www.futurescience.com/doi/suppl/10.2217/imt-2018-0118

\section{References}

1. Abdel-Rahman O, ElHalawani H, Fouad M. Risk of endocrine complications in cancer patients treated with immune check point inhibitors: a meta-analysis. Future Oncol. 12, 413-425 (2016).

2. Abdel-Rahman O, ElHalawani H, Fouad M. Risk of cutaneous toxicities in patients with solid tumors treated with immune checkpoint inhibitors: a meta-analysis. Future Oncol. 11, 2471-2484 (2015).

3. Abdel-Rahman O, ElHalawani H, Fouad M. Risk of gastrointestinal complications in cancer patients treated with immune checkpoint inhibitors: a meta-analysis. Immunotherapy 7, 1213-1227 (2015).

4. Heinzerling L et al. Cardiotoxicity associated with CTLA4 and PD1 blocking immunotherapy. J. Immunother. Cancer 4, 50 (2016).

5. Liberati A et al. The PRISMA statement for reporting systematic reviews and meta-analyses of studies that evaluate health care interventions: explanation and elaboration. PLoS Med. 6, e1000100 (2009).

6. Bersanelli M, Buti S. From targeting the tumor to targeting the immune system: transversal challenges in oncology with the inhibition of the PD-1/PD-L1 axis. World J. Clin. Oncol. 8, 37 (2017).

7. Higgins JP, Altman DG, Gøtzsche PC et al. The Cochrane Collaboration's tool for assessing risk of bias in randomised trials. BMJ 343, d5928 (2011).

8. Protocol Development | CTEP. https://ctep.cancer.gov/protocolDevelopment/electronic_applications/ctc.htm

9. Tierney JF, Stewart LA, Ghersi D, Burdett S, Sydes MR. Practical methods for incorporating summary time-to-event data into meta-analysis. Trials 8, 16 (2007).

10. Liu Z, Rich B, Hanley JA. Recovering the raw data behind a non-parametric survival curve. Syst. Rev. 3, 151 (2014).

11. DerSimonian R, Laird N. Meta-analysis in clinical trials. Control. Clin. Trials 7, 177-188 (1986).

12. Grant RL. Converting an odds ratio to a range of plausible relative risks for better communication of research findings. Bmj 348 , f 7450 (2014).

13. Kwon ED, Drake CG, Scher HI et al. Ipilimumab versus placebo after radiotherapy in patients with metastatic castration-resistant prostate cancer that had progressed after docetaxel chemotherapy (CA184-043): a multicentre, randomised, double-blind, Phase III trial. Lancet Oncol. 15, 700-712 (2014).

14. Borghaei H, Paz-Ares L, Horn L et al. Nivolumab versus docetaxel in advanced nonsquamous non-small-cell lung cancer. N. Engl. J. Med. 373, 1627-1639 (2015).

15. Eggermont AM, Chiarion-Sileni V, Grob JJ et al. Prolonged survival in stage III melanoma with ipilimumab adjuvant therapy. $N$. Engl. J. Med. 375, 1845-1855 (2016).

16. Fehrenbacher L Spira A, Ballinger $\mathrm{M}$ et al. Atezolizumab versus docetaxel for patients with previously treated non-small-cell lung cancer (POPLAR): a multicentre, open-label, Phase II randomised controlled trial. Lancet 387, 1837-1846 (2016).

17. Herbst RS, Baas P, Kim DW et al. Pembrolizumab versus docetaxel for previously treated, PD-L1-positive, advanced non-small-cell lung cancer (KEYNOTE-010): a randomised controlled trial. Lancet 387, 1540-1550 (2016).

18. Hodi FS, Lee $S$, McDermott DF et al. Ipilimumab plus sargramostim vs. ipilimumab alone for treatment of metastatic melanoma: a randomized clinical trial. JAMA 312, 1744-1753 (2014).

19. Hodi FS, Chesney J, Pavlick AC et al. Combined nivolumab and ipilimumab versus ipilimumab alone in patients with advanced melanoma: 2-year overall survival outcomes in a multicentre, randomised, controlled, Phase II trial. Lancet Oncol. 17, 1558-1568 (2016). 
20. Larkin J, Chiarion-Sileni V, Gonzalez R et al. Combined nivolumab and ipilimumab or monotherapy in untreated melanoma. $N$. Engl. J. Med. 2015, 23-34 (2015).

21. Ribas A, Kefford R, Marshall MA et al. Phase III randomized clinical trial comparing tremelimumab with standard-of-care chemotherapy in patients with advanced melanoma. J. Clin. Oncol. 31, 616-622 (2013).

22. Robert C, Long GV, Brady B et al. Nivolumab in previously untreated melanoma without BRAF mutation. N. Engl. J. Med. 372, 320-330 (2015).

23. Robert C, Schachter J, Long GV et al. Pembrolizumab versus ipilimumab in advanced melanoma. $N$. Engl. J. Med. 372, 2521-2532 (2015).

24. Brahmer J, Reckamp KL, Baas P et al. Nivolumab versus docetaxel in advanced squamous-cell non-small-cell lung cancer. $N$. Engl. J. Med. 373, 123-135 (2015)

25. Varricchi G, Galdiero MR, Marone G et al. Cardiotoxicity of immune checkpoint inhibitors. ESMO Open (2017). doi:10.1136/esmoopen-2017-000247.

26. Cardinale D, Sandri MT, Colombo A et al. Prognostic value of troponin I in cardiac risk stratification of cancer patients undergoing high-dose chemotherapy. Circulation 109, 2749-2754 (2004).

27. Cardinale D, Cipolla CM. Chemotherapy-induced cardiotoxicity: importance of early detection. Expert Rev. Cardiovasc. Ther. 14(12), 1297-1299 (2016).

28. Johnson DB, Balko JM, Compton ML et al. Fulminant myocarditis with combination immune checkpoint blockade. N. Engl. J. Med. 375, 1749-1755 (2016).

29. Heinzerling L, Ott PA, Hodi FS et al. Cardiotoxicity associated with CTLA4 and PD1 blocking immunotherapy. J. Immunother. Cancer 4(50), (2016). doi:10.1186/s40425-016-0152-y

30. Jain V, Bahia J, Mohebtash M, Barac A. Cardiovascular complications associated with novel cancer immunotherapies. Curr. Treat. Options Cardiovasc. Med. 19(36), (2017). doi:10.1007/s11936-017-0532-8

31. Freeman GJ, Long AJ, Iwai Y et al. Engagement of the Pd-1 immunoinhibitory receptor by a novel B7 family member leads to negative regulation of lymphocyte activation. J.Exp. Med. 192(7), 1027-1034 (2000).

32. Dong H, Zhu G, Tamada K, Chen L. B7-H1, a third member of the B7 family, co-stimulates T-cell proliferation and interleukin-10 secretion. Nat. Med.5(12), 1365-1369 (1999).

33. Grabie N, Gotsman I, DaCosta R et al. Endothelial programmed death-1 ligand 1 (PD-L1) regulates CD8+ T-cell-mediated injury in the heart. Circulation116(18), 2062-2071 (2007).

34. Lucas JA, Menke J, Rabacal WA, Schoen FJ, Sharpe AH, Kelley VR. Programmed death ligand 1 regulates a critical checkpoint for autoimmune myocarditis and pneumonitis in MRL mice. J. Immunol.181(4), 2513-2521 (2008).

35. Wang J, Okazaki IM, Yoshida T et al. PD-1 deficiency results in the development of fatal myocarditis in MRL mice. Int. Immunol. 22(6), 443-452 (2010).

36. Waterhouse P, Penninger JM, Timms E et al. Lymphoproliferative disorders with early lethality in mice deficient in Ctla-4. Science 270(5238), 985-988 (1995).

37. Tarrio ML, Grabie N, Bu D, Sharpe AH, Lichtman AH. PD-1 protects against inflammation and myocyte damage in T cell-mediated myocarditis. J. Immunol. 188(10), 4876-4884 (2012).

38. Baban B, Liu JY, Qin X, Weintraub NL, Mozaffari MS. Upregulation of programmed death-1 and its ligand in cardiac injury models: interaction with GADD153. PLoS ONE 10(4), e0124059 (2015). doi:10.1371/journal.pone.0124059

39. Rahouma M, Baudo M, Yahia $\mathrm{M}$ et al. Pneumonitis as a complication of immune system targeting drugs? Ameta-analysis of anti-PD/PD-L1 immunotherapy randomized clinical trials.. J. Thor. Dis.11(2), 521-534 (2019). 